\section{The Effect of Paricalcitol on Osteoprotegerin Production by Human Peripheral Blood Mononuclear Cells}

\section{To the Editor:}

It has been known for years that the ratio of receptor activator of nuclear factor- $\mathrm{\kappa B}$ ligand (RANKL) relative to its decoy receptor, osteoprotegerin (OPG), controls osteoclastogenesis. Under normal conditions the main source of RANKL and OPG is the osteoblast. Osteoblasts express on their surface RANKL that induces the formation of osteclasts from their precursors as well as their survival and activation. OPG is also produced and then secreted by the osteblasts, but it inhibits osteoclast formation by binding to RANKL and preventing binding to its receptor RANK on the surface of the osteoclasts' precursors ${ }^{1}$. However, a recent study suggests that both B and $\mathrm{T}$ lymphocytes also play a significant role in basal bone turnover, since $\mathrm{T}$ cells help B cells to produce $65 \%$ of total OPG in bone marrow in mice ${ }^{2}$. In inflammatory diseases the participation of activated lymphocytes in pathological bone erosion is established ${ }^{3}$.

Vitamin D promotes osteoclastogenesis through RANKL upregulation and OPG downregulation in the osteoblasts ${ }^{4,5}$. In vitro experiments, in the absence of inflammatory stimuli, have shown that 1,25-dihydroxyvitamin $\mathrm{D}_{3}$ initially downregulates OPG by accelerating the degradation of OPG mRNA and by transrepressing the OPG gene through its activating protein1 (AP-1) binding site. But later the OPG gene becomes insensitive to 1,25-dihydroxyvitamin $\mathrm{D}_{3}$ and reverts to its steady-state expression level over time ${ }^{4}$. Interestingly, under inflammatory conditions, inhibition of OPG production is more stable. Inflammatory cytokines such as interleukin $1 \beta$ (IL-1ß), IL-6, IL-11, IL-17, and tumor necrosis factor- $\alpha$ (TNF- $\alpha$ ) stimulate osteoclast development by increasing the expression of RANKL in the presence of 1,25-dihydroxyvitamin $D_{3}$, whereas these cytokines further suppressed OPG production mediated by 1,25-dihydroxyvitamin $\mathrm{D}_{3}{ }^{5}$. Importantly, experimental data and clinical observations confirmed that vitamin $\mathrm{D}$ has immunomodulatory/antiinflammatory properties ${ }^{6}$. Epidemiological studies have shown an inverse relation between serum levels of vitamin D and the occurrence or severity of various inflammatory bone erosive diseases. Rheumatoid arthritis is the best example ${ }^{7,8}$.

Under inflammatory conditions vitamin D exerts osteolysis in part due to downregulation of OPG. Recently, we have shown that the vitamin D analog paricalcitol has antiinflammatory properties, since it reduces both basal and lipopolysaccharide (LPS)-induced TNF- $\alpha$ and IL- 8 production by the peripheral blood mononuclear cells (PBMC) ${ }^{9}$. Additionally, paricalcitol is less calcemic than vitamin $\mathrm{D}^{10}$, which allows its administration in higher doses. In this context, its use for attenuating inflammation in chronic inflammatory diseases could be limited by its possible detrimental osteolytic effect on bone. Therefore, and considering the participation of activated lymphocytes in pathological bone erosion ${ }^{3}$, it is of interest to assess the effect of paricalcitol on OPG production by PBMC under inflammatory conditions.

In brief, 10 healthy volunteers ( 5 men and 5 women, mean age $40.3 \pm$ $9.36 \mathrm{yrs}$ ) were enrolled into our study. Informed consent was obtained from each individual and the hospital ethics committee gave its approval to the study protocol. PBMC at a concentration of $10^{6}$ cells per well were cultured for $48 \mathrm{~h}$ in the presence or not of LPS as an inflammatory stimulus (Sigma-Aldrich, St. Louis, MO, USA) at a concentration of $100 \mathrm{ng} / \mathrm{ml}$ and in the presence or not of paricalcitol (Zemplar, Abbott Laboratories, North Chicago, IL, USA) at a concentration of $10^{-8} \mathrm{M}$. OPG was measured in the supernatants with ELISA (Bender MedSystems, Vienna, Austria). The normal distribution of the variables was confirmed using the 1-sample Kolmogorov-Smirnov test, and for comparison of means paired t-test was used.

Basal OPG concentration was decreased by paricalcitol $(79.00 \pm 25.87$ $\mathrm{pg} / \mathrm{ml}$ vs $52.40 \pm 24.97 \mathrm{pg} / \mathrm{ml} ; \mathrm{p}<0.001)$. LPS decreased OPG concentration $(79.00 \pm 25.87 \mathrm{pg} / \mathrm{ml}$ vs $56.80 \pm 27.16 \mathrm{pg} / \mathrm{ml} ; \mathrm{p}<0.001)$ as well. Paricalcitol failed to decrease further the OPG concentration in LPS-treated PBMC $(56.80 \pm 27.16 \mathrm{pg} / \mathrm{ml}$ vs $62.10 \pm 26.38 \mathrm{pg} / \mathrm{ml}$; $\mathrm{p}=0.447)$.
In clinical practice, the main obstacle for vitamin $\mathrm{D}$ administration as an immunomodulatory/antiinflammatory agent is that at high doses it provokes hypercalcemia. Paricalcitol is a vitamin D analog that is safe, since it has been used for years in the treatment of secondary hyperparathyroidism in hemodialysis patients ${ }^{11}$ and, importantly, it is less calcemic ${ }^{10}$. It has antiinflammatory properties as well ${ }^{9}$. In our study, paricalcitol did not decrease OPG production by the LPS-treated PBMC, indicating that under inflammatory conditions paricalcitol may not promote osteoclastogenesis. This feature of paricalcitol, along with its antiinflammatory properties and the less calcemic action, indicate that paricalcitol may have an advantage over vitamin D regarding its possible therapeutic role in inflammatory diseases characterized by pathological bone erosion.

THEODOROS ELEFTHERIADIS, MD, PhD, Research Institute, Theagenion Anticancer Hospital, Thessaloniki, and Department of Nephrology, General Hospital of Serres, Serres; GEORGIA ANTONIADI, MD, PhD, Department of Nephrology, General Hospital of Serres; VASSILIOS LIAKOPOULOS, MD, PhD, Department of Nephrology, University Hospital of Thessaly, Larissa; GRAMMATI GALAKTIDOU, $\mathrm{PhD}$, Research Institute, Theagenion Anticancer Hospital, Thessaloniki, Greece. Address reprint requests to Dr. T. Eleftheriadis, Department of Nephrology, General Hospital of Serres, 3rd km Serres-Drama, 62100 Serres, Greece. E-mail: teleftheriadis@yahoo.com

\section{REFERENCES}

1. Boyce BF, Xing L. Biology of RANK, RANKL, and osteoprotegerin. Arthritis Res Ther 2007;9 Suppl:S1-S7.

2. Li Y, Toraldo G, Li A, et al. B cells and T cells are critical for the preservation of bone homeostasis and attainment of peak bone mass in vivo. Blood 2007;109:3839-48.

3. Nakashima T, Takayanagi $\mathrm{H}$. The dynamic interplay between osteoclasts and the immune system. Arch Biochem Biophys 2008;473:166-71

4. Kondo T, Kitazawa R, Maeda S, Kitazawa S. 1 alpha,25 dihydroxyvitamin D3 rapidly regulates the mouse osteoprotegerin gene through dual pathways. J Bone Miner Res 2004;19:1411-9.

5. Nakashima T, Kobayashi Y, Yamasaki S, et al. Protein expression and functional difference of membrane-bound and soluble receptor activator of NF-kB ligand: modulation of the expression by osteotropic factors and cytokines. Biochem Biophys Res Commun 2000;275:768-75

6. van Etten E, Mathieu C. Immunoregulation by 1,25-dihydroxyvitamin D3: basic concepts. J Steroid Biochem Mol Biol 2005;97:93-101.

7. Cutolo M, Otsa K, Laas K, et al. Circannual vitamin D serum levels and disease activity in rheumatoid arthritis: Northern versus Southern Europe. Clin Exp Rheumatol 2006;24:702-4.

8. Cutolo M, Otsa K, Uprus M, Paolino S, Seriolo B. Vitamin D in rheumatoid arthritis. Autoimmun Rev 2007;7:59-64.

9. Eleftheriadis T, Liakopoulos V, Antoniadi G, Stefanidis I, Galaktidou G. Paricalcitol at clinically achievable concentration decreases basal and LPS induced TNF-a and IL-8 production by human peripheral blood mononuclear cell [abstract]. Abstract book, 7th Annual Conference on Prevention in Renal Disease, Toronto, Canada, September 19-20, 2008: 8.

10. Llach F, Yudd M. Paricalcitol in dialysis patients with calcitriol resistant secondary hyperparathyroidism. Am J Kidney Dis 38;5 Suppl:S45-50.

11. Lindberg J, Martin KJ, Gonzalez EA, Acchiardo SR, Valdin JR, Soltanek C. A long-term, multicenter study of the efficacy and safety of paricalcitol in end-stage renal disease. Clin Nephrol $2001 ; 56: 315-23$.

J Rheumatol 2009;36:4;doi:10.3899/jrheum.080987 